\title{
Antibacterial activity of Glycyrrhiza glabra roots against certain gram-positive and gram-negative bacterial strains
}

\author{
A parajita Gupta*, D. K. M aheshwari ${ }^{1}$ and G. K handelwal ${ }^{1}$ \\ Department of Microbiology, Kanya Gurukula Mahavidyalaya, Gurukul Kangri Vishwavidyalaya, Haridwar- 249404 \\ (Uttarakhand), INDIA \\ ${ }^{1}$ Department of Botany and Microbiology, Gurukula Kangri Vishwavidyalaya, Haridwar- 249404 (Uttarakhand), INDIA \\ *Corresponding author. E-mail: aparajitag88@gmail.com
}

Received: September 19, 2013; Revised received: November 10, 2013; Accepted: N ovember 20, 2013

\begin{abstract}
The present study aimed to evaluate the antibacterial potency of grinded crude material (root of Glycyrrhiza glabra) against some gram-positive and gram-negative bacterial strains. Two solvents (methanol and acetone) were used to extract the phytochemicals from the test material. Four different concentrations $100 \%, 75 \%, 50 \%$ and $25 \%$ ) of methanolic and acetonic extract were used to investigate the inhibiting properties against Salmonella typhi, Escherichia.coli, Vibrio cholerae, Staphylococcus aureus, Bacillus cereus and Bacillus subtilis strains. Among methanol and acetone extracts, later exhibited low antibacterial activity. The $100 \%(\mathrm{w} / \mathrm{v})$ concentration of both extracts showed maximum inhibition against B. subtilis followed by E. coli, S. aureus, B. cereus, S. typhi and V. Cholerae. Maximum activity in acetonic extract was obtained against B. cereus followed by S. typhi, E. coli, V. cholerae and $\mathrm{S}$.aureus and minimum in $\mathrm{B}$. subtilis. A reverse pattern of inhibition activity was found in both extacts (methanolic and acetonic) against B. subtilis. Maximum activity was found in methanolic extract against B. subtilis $(18.6 \mathrm{~mm})$ but it was only $14.3 \mathrm{~mm}$ against this strain in acetonic extract. The antibacterial activity of the crude samples corresponded to that of concentration. Hence there was positive correlation of antibacterial activity with the test material.
\end{abstract}

Keywords: Antibacterial actvity, Glycyrhiza glabra, Gram-positive bacteria, Gram-negative bacteria

\section{INTRODUCTION}

Today, the increasing failure of chemotherapeutic and antibiotics resistance exhibited by microorganisms have been a major problem and this leads to the screening of medicinal plants for their potential for antimicrobial activity (Laxmi et al., 2011). In general antimicrobial nature of the drug plants is due to the secondary metabolite in the form of generation of new chemical component some of them are highly effective against certain pathogenic and they can be exploited for their industrial applications. As the G. glabra has been used since ancient times as a folk medicine and still has its more significance in indigenous system of medicine. Its root contains commercially important chemical called licorice. The chemicals components responsible for antioxidant and antibacterial activity present in Glycyrrhiza glabra root have been reported such as Glycyrrhizin, Glycyrrhizinic acid etc (Tang and Eisenbrand., 1992); glabridin, glabrene, glabrol, licoflavonol, glycyrol, licoricone, formononetin, phaseollinisoflavan, hispaglabridin AandB, 3hydroxyglabrol, 3'-methoxyglabridin (Kinoshita et al., 1976; Mitscher et al., 1978, 1980; Saitoh etal., 1978; Fukai et al., 1996, 2002a,b, 2003; Glenn et al., 2005); glabranin isomer, narigenin, lupiwightenone (Biondi et al., 2003,
2005). Therefore, it is aimed to turn our investigations to natural products from G. glabra roots for antibacterial potential so as to develop new drug molecule. The bacterial strains used in the present study are such as $B$. subtilis, B. cereus, E. coli, S. typhi, S. aureus, V. cholerae. B. subtilis is a gram-positive and rod shaped bacterium, which has the ability to form a tough, protective endospore, allowing the organisms to tolerate extreme environmental conditions. B. cereus is an endemic, soil dwelling, gram-positive, rod shaped beta haemolytic bacterium. It is the cause of "Fried Rice Syndrome" as the bacteria is classically contracted from fried rice dishes that have been kept at room temperature for hours (Glenn et al., 2005). E. coli is a gram-negative, rod shaped bacterium found in lower intestine of warm blooded organisms (endotherms). Virulent strains of E. coli can cause gastroenteritis, urinary tract infections and neonatal meningitis. S. aureus is a facultative anaerobic gram-positive coccal bacterium also known as "golden staph". S. aureus is frequently found in human respiratory tract and on skin. It is the common cause of skin infections (e.g. boils) and food poisoning. V. cholerae is gram-negative comma shaped bacterium. V. cholerae secretes cholera toxin, a protein that cause profuse, 
watery diarrhoea during the course of infection. Sweetness of G. glabra root is mainly due to presence of glycyrrhizin component. Glycyrrhiza glabra L. has been used since long in indigenous medicine system either alone or with the combination of other drug plant materials. The antimicrobial activity of $\mathrm{G}$. glabra against $M$ ycobacterium tuberculosis has been established (Gupta et al., 2008).

Licorice extracts have been used for more than 60 years in Japan to treat chronic hepatitis and also have therapeutic benefits against other viruses, including human immunodeficiency virus (HIV), cytomegalovirus (CMV) and Herpes simplex. Fukai et al. (2002a, b) reported the Anti-Helicobacter pylori and antibacterial activities of flavonoids from licorice extracts. The present study was undertaken to evaluate the antibacterial activity of G. glabra against gram- positive and gram- negative bacterial strains.

\section{MATERIALS AND METHODS}

Plant material: The roots of G. glabra were procured locally from Hansa Pharmacy, located in Prem Nagar Ashram, Haridwar. The roots were washed with sterilized distilled water, shed hot air dried and then grind into coarse powder under sterilized condition. The grinded powder was packed in sterilized poly bags than kept at room temperature in research laboratory.

Test organisms/ bacterial strains: Pure cultures of B. cereus (MTCC 6728), S. typhi (MTCC 3216), S. aureus (MTCC 7443), V. cholerae (MTCC 3904), B. subtilis (MTCC 441) were obtained from microbial type culture collection centre of institute of microbial technology (IMTECH) Chandigarh, India and E. coli from SGPGI Lucknow. All the organisms were subcultured on nutrient agar medium (NAM), V. cholerae was subcultured on Luria Bertani Agar (LBA) and incubated at $37 \pm 1{ }^{\circ} \mathrm{C}$ for 24 hours and preserved at low temperature.

Preparation of root extract: The solvents used for the extraction procedure were acetone and methanol of analytical grades. These two solvents already have been reported as excellent solvent for extraction of maximum phytochemicals of G. glabra species and for high performance liquid chromatography i.e. HPLC (Gatto et al., 2002). Further ethyl alcohol and other solvents also have been used by Meena et al., 2010. After weighing $125 \mathrm{gm}$ of grinded root powder it was extracted using $500 \mathrm{ml}$ of the individual solvent $(25 \% \mathrm{w} / \mathrm{v})$ for $24 \mathrm{hrs}$ in soxhlet extracter and was then subjected to filtration through filter paper (Whatman No.1). The solvent was allowed to evaporate under controlled temperature to get the volume of $125 \mathrm{ml}$. The final extract was so further dried to obtain $1.0 \mathrm{ml}$ of extract solution represented $1.0 \mathrm{gm}$ of powdered material. The obtained extract was $100 \%$ concentrated and was used as the stock solution which was further diluted to prepare $75 \%, 50 \%$ and $25 \%$ concentration of the extract. Methanol and acetone $(100 \%)$ were used as control.

Antibacterial activity: The antibacterial activity of G. Glabra was tested by Well-agar diffusion method. About $100 \mu 1$ of standardized microbial stock suspension $\left(1 \times 10^{5} \mathrm{cfu} / \mathrm{ml}\right)$ of $24 \mathrm{hrs}$ old cultures of test organism was thoroughly mixed with melted nutrient agar medium (NAM) and poured into sterile petriplates. Five wells of $6 \mathrm{~mm}$ diameter were made in agar medium using sterile borer and filled with $100 \mu 1$ of each of the extract concentration. All the solvents served as negative control. Zones of inhibition obtained around well was measured after an incubation period of 24 hours at $37^{\circ} \mathrm{C}$ was used as positive control.

Phytochemical analysis by high performance liquid chromatography (HPLC): HPLC has ability to separate and identify the compounds present in any specific sample that can be dissolved in a liquid in trace concentrations as low as parts per trillion. Due to this versatility this is being used in pharmaceutical industry. Therefore, in the present investigation, phytochemical analysis of G. glabra of methanolic and acetonic extract was investigated by HPLC. It is the advanced form of Column chromatography that pumps sample mixture/ analytes in a solvent at high pressure through column with chromatographic packing material. The column of HPLC used was made up of stainless steel.

\section{RESULTS AND DISCUSSION}

In the present study, maximum effective inhibition in methanolic extract at $100 \%$ was found against $B$. subtilis $(18.6 \mathrm{~mm})$ followed by E . coli $(18.3 \mathrm{~mm})$, S. aureus, B . cereus $(17.6 \mathrm{~mm})$ and $\mathrm{S}$. typhi $(16.3 \mathrm{~mm})$, whereby the minimum inhibition zone was recorded against $\mathrm{V}$. cholerae. Acetonic extract did not show same trend of antimicrobial activity of bacterial strains as were found in methanolic extract. Maximum effective inhibition in $100 \%$ acetonic drug extract was recorded against $B$. cer eus $(16.3 \mathrm{~mm})$ followed by S. typhi $(16.0 \mathrm{~mm})$, in E . coli $(15.3$ $\mathrm{mm})$, in V. cholerae and S. aureus (15.0 $\mathrm{mm}$ ), whereas the minimum inhibition zone was (14.3) recorded against $B$. subtilis. In general declined effective pattern of inhibition zone with dilution was recorded in all dilutions of both extract (Table 1). Makky et al. (2012) studied the phytochemicals and their role in antimicrobial activity of six drug plants including G. glabra against antibiotic resistance bacterial strain (ARB) isolated from pharmaceutical product and from hospital. The methanolic extract of G. glabra showed antibacterial activity against both gram-positive and gram-negative antibiotic resistant bacterial strains (ARB) isolated from pharmaceutical product i.e Alcalignes xylosoxidans (UNO9D) and Staphylococcus xylosus (ASP13D). Gupta 

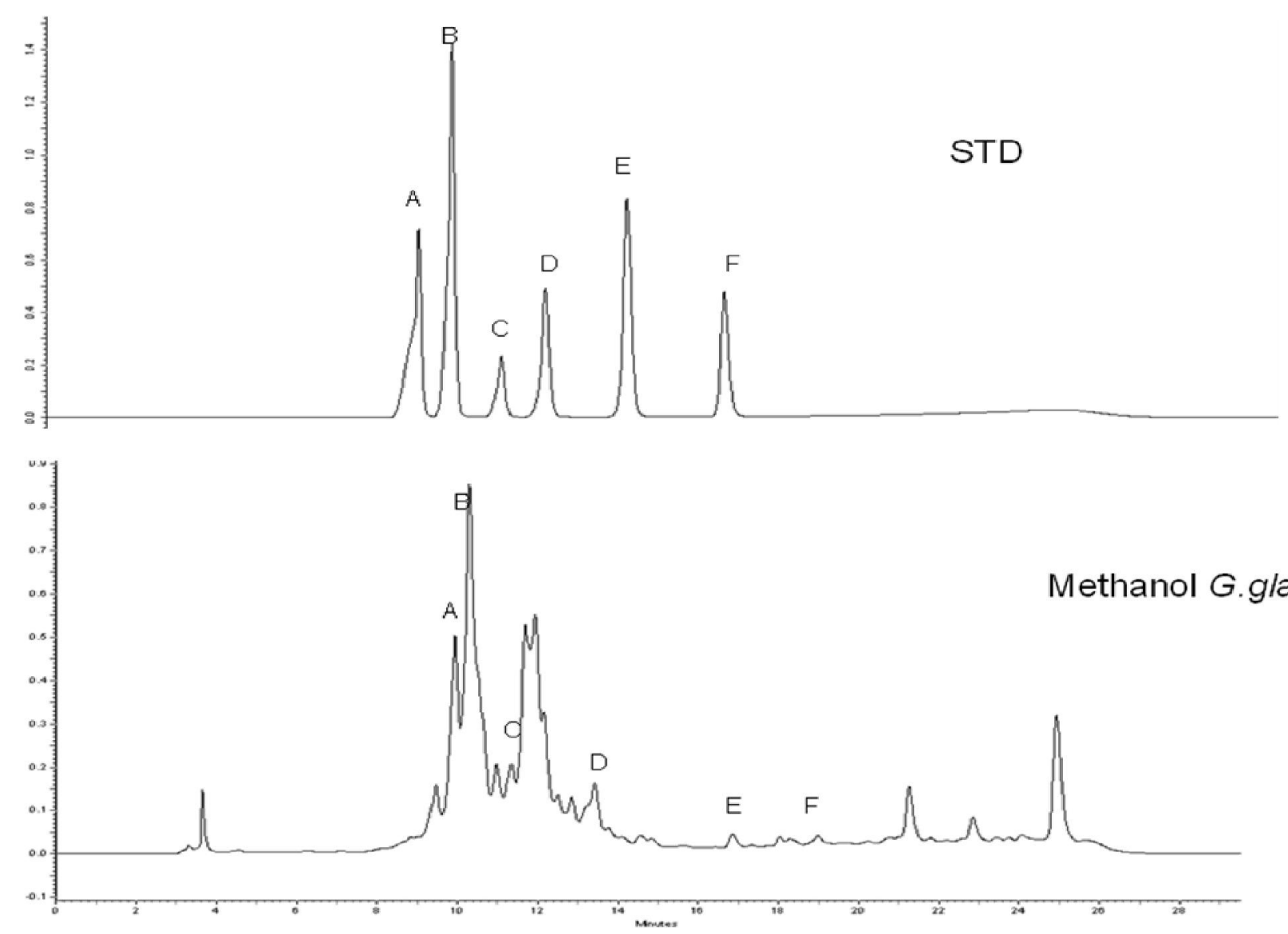

Fig. 1. Methanolic extract of G. glabra. A-Chlorogenic acid; B-Caffeic acid; C-Rutin; D-M ycricitin; E-Q uercetin; F-K aempferol; STD-standard.
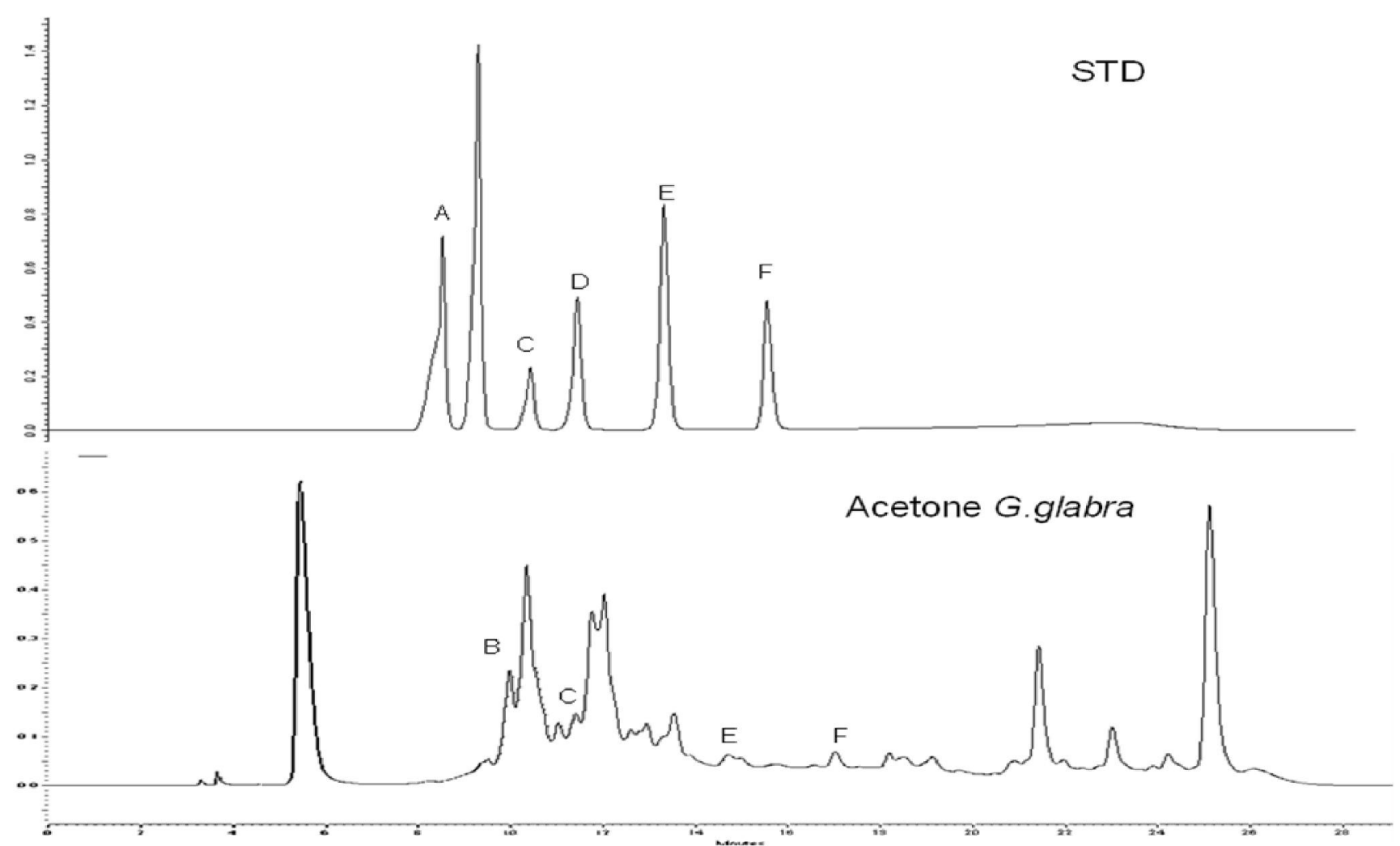

Fig. 2. Acetonic extract of G. glabra. A-Chlorogenic acid; B-Caffeic acid; C-Rutin; D-M ycricitin; E-Quercetin; F-Kaempferol; STD - standard. 
Table 1. Antibacterial activity of methanolic and acetonic extract of G. glabra (Mean \pm SD of three replicates).

\begin{tabular}{|c|c|c|c|}
\hline \multirow[t]{2}{*}{ Test organisms } & \multirow{2}{*}{ Extract concentration (\%) } & \multicolumn{2}{|c|}{ Effective inhibition zone (mm) } \\
\hline & & M ethanol & A cetone \\
\hline Control (methanol/acetone) & Absolute (100\%) & -- & -- \\
\hline \multirow[t]{4}{*}{ S. typhi } & 100 & $16.3^{* *} \pm 0.57$ & $16.0^{* *} \pm 1.0$ \\
\hline & 75 & $14.3^{* * *} \pm 0.57$ & $13.3^{* *} \pm 0.57$ \\
\hline & 50 & $13.0^{* *} \pm 1$ & $11.6^{* *} \pm 0.57$ \\
\hline & 25 & $12.3^{* *} \pm 0.57$ & $10.3^{* *} \pm 0.57$ \\
\hline \multirow[t]{4}{*}{ E. coli } & 100 & $18.3^{* *} \pm 1.15$ & $15.3^{* *} \pm 1.15$ \\
\hline & 75 & $15.0^{* *} \pm 0$ & $13.6^{* *} \pm 0.57$ \\
\hline & 50 & $13.6^{* *} \pm 0.57$ & $12.0^{* *} \pm 1.0$ \\
\hline & 25 & $11.3^{* * *} \pm 0.57$ & $10.0^{* * *} \pm 0.0$ \\
\hline \multirow[t]{4}{*}{ V. cholerae } & 100 & $15.3^{* * *} \pm 1.15$ & $15.0^{* *} \pm 1.0$ \\
\hline & 75 & $12.3^{* * *} \pm 0.57$ & $12.6^{* *} \pm 0.57$ \\
\hline & 50 & $10.6^{* *} \pm 1.15$ & $11.3^{* *} \pm 0.57$ \\
\hline & 25 & $8.3^{* *} \pm 0.57$ & $6.3^{* *} \pm 1.52$ \\
\hline \multirow[t]{4}{*}{ S. aureus } & 100 & $17.6^{* * *} \pm 1.15$ & $15.0^{* *} \pm 1.0$ \\
\hline & 75 & $15.3^{* * *} \pm 0.57$ & $12.6^{* *} \pm 0.57$ \\
\hline & 50 & $13.6^{* * *} \pm 0.57$ & $11.3^{* *} \pm 1.15$ \\
\hline & 25 & $12.0^{* *} \pm 0$ & $9.3^{* *} \pm 0.57$ \\
\hline \multirow[t]{4}{*}{ B. cereus } & 100 & $17.6^{* *} \pm 1.52$ & $16.3^{* *} \pm 1.52$ \\
\hline & 75 & $14.3^{* *} \pm 0.57$ & $14.6^{* *} \pm 1.15$ \\
\hline & 50 & $13.6^{* *} \pm 0.57$ & $13.0^{* *} \pm 1.0$ \\
\hline & 25 & $12.6^{* *} \pm 0.57$ & $11.0^{* * *} \pm 1.0$ \\
\hline \multirow[t]{4}{*}{ B. subtilis } & 100 & $18.6^{* * *} \pm 0.57$ & $14.3^{* *} \pm 0.57$ \\
\hline & 75 & $16.3^{* * *} \pm 0.57$ & $13.6^{* *} \pm 0.57$ \\
\hline & 50 & $14.0^{* *} \pm 0$ & $12.3^{* *} \pm 0.57$ \\
\hline & 25 & $12.6^{* *} \pm 0.57$ & $9.6^{* *} \pm 0.57$ \\
\hline
\end{tabular}

Well size in each case: $6 \mathrm{~mm}$; ** Well size in each case: $6 \mathrm{~mm}$; significantly different at $1 \%$ level of ANOVA, (一): No activity.

Table 2. Phytochemical analysis of G. glabra.

\begin{tabular}{lcccccc}
\hline Extract type & \multicolumn{5}{c}{ Q uantification on percent dry weight basis of differ ent compounds } \\
\cline { 2 - 7 } Methanol & Chlorogenic acid & Caffeic acid & Rutin & M yricitin & Quercetin & K aempferol \\
\cline { 2 - 7 } Acetone & 0.014008 & 0.062226 & 0.05312 & 0.016671 & 0.003467 & 0.007102 \\
& ND & 0.034316 & 0.087007 & ND & 0.006814 & 0.013876 \\
\hline
\end{tabular}

(ND: Not detected)

et al. (2008) have also found the antibacterial activity of G. glabra against M ycobacterium tuberculans.

Trend of variant behaviour of different crude extract against all test bacterial strains may be due to the presence or absence as well as quantitative variations of chemicals responsible for bioefficacy. Drug efficacy loss in term of reduction of inhibition zone in mm in last dilution i.e $25 \%$ drug concentration in comparison of $100 \%$ concentration in methanolic extract was recorded i.e 4.0 mm against $S$. typhi, $7.0 \mathrm{~mm}$ against $E$. coli, $7.0 \mathrm{~mm}$ against V. cholerae, $5.6 \mathrm{~mm}$ against $S$. aureus, $5.0 \mathrm{~mm}$ against $B$. cereus and $6.0 \mathrm{~mm}$ against $B$. subtilis. In acetonic extract total decreased value is $5.7 \mathrm{~mm}$ against $\mathrm{S}$. typhi, $5.3 \mathrm{~mm}$ against $E$. coli, 8.7 mm against $V$. cholerae, 5.7 mm against S. aureus, 5.3 mmagainst $B$. cer eus and $4.7 \mathrm{~mm}$ against $B$. subtilis was recorded. Total loss of drug potency at $25 \%$ concentration in comparison of $100 \%$ of each specific crude drug extract against specific bacterial strains may be either due to maximum dilution or biologically active compounds which were present in very small traces and were reduced surely responsible for the much loss of the bioefficacy. But the potency loss ratio was quiet different against test strains among different crude extract. A definite ratio of reduction of extract potency in term of reduction of inhibition zone at last concentration could not be observed. In methanolic extracts, maximum loss 
of drug potency was found in E. coli and V. cholerae (7 $\mathrm{mm})$ followed by $B$. subtilis $(6 \mathrm{~mm})$ and lowest value was found against B.cereus. In acetonic extract maximum reduction of drug plant was found against $\mathrm{V}$. Cholerae i.e $8.7 \mathrm{~mm}$ followed by S.aureus $5.7 \mathrm{~mm}$ and minimum against B. subtilis i.e., $4.9 \mathrm{~mm}$.

Results of phytochemical analysis based on HPLC revealed that both methanolic and acetonic extracts showed variation in terms of absence or presence of certain compounds as well as in their quantification on percent dry weight basis of different compounds. Two components i.e. chlorogenic acid and myricitin were not found in acetonic crude extract of G. Glabra (Figs.1, 2). In general lowered value bioefficacy was recorded in acetonic extract (Table 1) may be due to the absence of certain effective biological components as it is evident by Table 2 in which chlorogenic acid and myricitin were absent and possibility cannot be ignored about involvement of these two in the bioefficacy. Chlorogenic acid compound also possesses inhibitory effect on tumor formation in mice showed anti cancerous property (MouTuan Huang et al., 1988). Fukai et al. (2002 a) reported certain flavonoids for licorice such as glabridin, glabrene, licochalcone A, licoisoflavone $\mathrm{B}$ which showed antibacterial activity against drug resistant $H$. pylori. Fukai et al. (2002 b) further reported antibacterial activity of flavonoid against methicillin resistant strain of $\mathrm{S}$. aureus.

Caffeic acid outperformed the other antioxidants reducing aflatoxin production by more than $95 \%$. These studies are the first to show that the oxidative stress that would otherwise trigger or enhance Aspergillus flavus aflatoxin production can be stymied by caffeic acid. This opens the door to using natural fungicide methods by supplementing trees with anitoxidants.

Chlorogenic acid is reported to be a chemical sensitizer responsible for human respiratory allergy to certain types of plant material (Freedman et al., 1964). Quercitin seems to exert antibacterial activity against almost all the strains of bacteria known to cause respiratory, gastrointestinal, skin and urinary disorders (Rigano et al., 2007). Quercitin appeared active against different viruses (Kaul et al., 1985) including HIV (Mahmood et al., 1996), probably due to inhibition of reverse transcriptase (Nakane and Ono 1990).

\section{Conclusion}

It was concluded that both methanolic /acetonic) extracts of G. glabra had potential in in vitro antibacterial activity against all the studied gram-positive and gram-negative bacterial strains. However, the study needs evaluation of various components (chlorogenic acid, caffeic acid, quercitin, myricitin, kaempferol, rutin) detected in the ethanolic and methanolic extracts for the antibacterial activity. As the extracts obtained are of plant origin, therefore it would be safer than modern medicines in terms of side-effects for their use in antibacterial activity of various microbes.

\section{REFERENCES}

Biondi, D.M., Rocco, C. and Ruberto, G. (2005). Dihydrostillbene derivatives from G lycyrrhiza glabra leaves. J Nat Prod, 68:1099-1102

Biondi, D.M., Rocco, C and Ruberto, G. (2003). New Dihydrostillbene derivatives from leaves of Glycyrrhiza glabra and evaluation of their antioxidant activity. J ournal of Natural Products, 66:477-480.

Freedman, Samuel O., Shulman, Robert., Krupey, John and Sehon, A.H. (1964). Antigenic properties of chlorogenic acid . J ournal of allergy, 35(2):97

Fukai, T., Tantai, L and Nomura, T. (1996). Isoprenoid substitute flavonoid from Glycyrrhiza glabra. Phytochemistry, 43:531-532.

Fukai,T., Ali, M., Kaitou, K., Kanda, T., Terada, S and Nomura, T. (2002a). Anti-Helicobacter pylori flavonoid from licorice extract. Life Sciences, 71:1449-1463.

Fukai, T., Marumo, A., Kaitou, K., Kanda, T., Terada, S and Nomura T. (2002b). Antimicrobial activity of licorice flavonoids against methicillin-resistant Staphylococcus aureus. Fitoterapia, 73: 536-539.

Fukai, T., Satoh, K., Nomura, T., Sakagami, H. (2003). Preliminary evaluations of antinephritis and radical scavenging activities of glabridin from G. glabra. Fitoterapia, 74: 624-629.

Gatto, Maria Teresa., Serena Falcocchio., Eleonora Grippa., Gabriela Mazzanti., Lucia Battinelli., Giovanni Nicolosi., Daniela Lambusta and Luciano saso. (2002).Antimicrobial and Antilipase activity of quercitin and its $\mathrm{C} 2-\mathrm{C} 16$ 3-OAcyl-Esters. J ournal of bioorganic and medicinal chemistry, 10:269-272.

Glenn, Asaeda., Caicedow, Gilbert and Swanson, Christopher (2005). Fried Rice Syndrome.J ournal of Emergency M edical Services, 30 (12): 30-32

Gupta, V.K., Fatima, A., Faridi, U., Negi, A.S., Shanker, K., Kumar, J.K., Rahuja, N., Luqman, S., Sisodia, B.S., Saikia D., Darokar, M.P. and Khanuja, S.P.S. (2008). Antimicrobial potency of Glycyrrhizaglabra roots. Journal of Ethnopharmacology, 116: 377-380

Kaul, T.N., Middleton Jr, E and Ogra, P.L. (1985). Antiviral effect of flavonoids on human viruses. J. Med. Virol, 115 , 71-79.

Kinoshita, T., Saitoh, T and Shibata, S. (1976). Flavonols of licorice root. Chem Pharma Bull, 24: 991-994.

Laxmi, Ahirwal., Singh, Siddhartha and Mehta, Archana (2011). Antimicrobial Screening of Methanol and Aqueous extracts of SwertiaC hirata. Int J PharmaSci, 3: 142-146.

Mahmood, N., Piacente, S., Pizza, C., Burke, A., Khan, A.I and Hay, A.J. (1996). Biochem. Biophys. Res. Commun. $229: 73$

Makky, E.A., Yusoff Mashitah, M and Ibrahim M.M. (2012). Impact of medicinal plants phytocomponents against antibiotic resistant bacteria. J ournal of Chemical and Pharmaceutical Research, 4(1): 881-893.

Meena, Ajay Kumar., Singh, Arjun., Sharma, Kiran., Kumari, Suman and Rao M.M. (2010). Physicochemical and 
preliminary phytochemical studies on the rhizome of G lycyrrhiza glabra. Int. J of Pharmacy and Pharmaceutical Science, 02:48-50.

Mitscher, L.A., Park, S., Omota, S., Clark, G.W. and Clark, D. (1978). Antimicrobial agents from higher plants, G lycyrrhiza glabra L. I. some antimicrobial isoflavans, isoflavenes, flavanones and isoflavones. Heterocycles, 9: 1533-1537.

Mitscher, L.A., Park, Y.H., Clark, D and Beal, J.L. (1980). Antimicrobial agents from higher plants, Antimicrobial, isoflavanoids and related substances from Glycyrrhiza glabra L. var. typical. J ournal of Natural Products, 43 : 259-269.

Mou-Tuan Huang., Robert C. Smart. and Ching-Quo Wong. (1988). Inhibitory Effect of curcumin, chlorogenic acid, caffeic acid and ferulic acid on tumor promotion in mouse skin by 12-o-Tetradecanoylphorbol-13-acetate. American Aassociation for Cancer Research, 48: 5941-5946.

Nakane, H. and Ono, K. (1990). Mechanism of inhibition of various cellular DNA and RNA polymerases by several flavonoids. J. Biochem.,108(4):609-13.

Rigano, D., Formisano, C., Basile, A., Lavitol, A., Senatore, F., Rossellis, S and Bruno, M. (2007). Antibacterial activity of flavonoids and phenyl propanoids from Marrubium globosum ssp. Libanoticum. Phytother Res,21(4): 395-7.

Saitoh, T., Noguchi, H and Shibata, S. (1978). A new isoflavone and the corresponding isoflavanone of licorice root. Chem Pharma Bull, 26: 144-147.

Tang, W and Eisenbrand G. (1992). Chinese drugs of Plant Origin. Springer-Verlag, B erlin, Germany; pp: 567-588. 